\title{
Utility of Synechocystis sp. PCC 6803 glutaredoxin A as a platform to study high-resolution mutagenesis of proteins
}

\author{
David B. Knaff ${ }^{1,2}$ and Roger B. Sutton ${ }^{3,4 *}$ \\ ${ }^{1}$ Department of Chemistry and Biochemistry, Texas Tech University, Lubbock, TX, USA \\ ${ }^{2}$ Center for Biotechnology and Genomics, Texas Tech University, Lubbock, TX, USA \\ ${ }^{3}$ Department of Cell Physiology and Molecular Biophysics, Texas Tech University Health Sciences Center, Lubbock, TX, USA \\ ${ }^{4}$ Center for Membrane Protein Research, Texas Tech University Health Sciences Center, Lubbock, TX, USA
}

\section{Edited by:}

Jose A. Traverso, Consejo Superior de Investigaciones Científicas, Spain

\section{Reviewed by:}

Nathan Nelson, Tel-Aviv University, Israel

Xiaoqiang Wang, Samuel Roberts

Noble Foundation, USA

Javier Lopez-Jaramillo, Universidad de Granada, Spain

\section{*Correspondence:} Physiology and Molecular

Biophysics, Texas Tech University Health Sciences Center, 3601 4th St., Mailstop 6551, Lubbock, TX 79430-6551, USA

e-mail: roger.b.sutton@ttuhsc.edu
Roger B. Sutton, Department of Cell

Glutaredoxin from the cyanobacterium Synechocystis sp. PCC 6803 is a small protein, containing only 88 amino acids, that participates in a large number of redox reactions, serving both as an electron donor for enzyme-catalyzed reductions and as a regulator of diverse metabolic pathways. The crystal structures of glutaredoxins from several species have been solved, including the glutaredoxin A isoform from the cyanobacterium Synechocystis sp. PCC 6803. We have utilized the small size of Synechocystis glutaredoxin $A$ and its propensity to form protein crystals that diffract to high resolution to explore a long-standing question in biochemistry; i.e., what are the effects of mutations on protein structure and function? Taking advantage of these properties, we have initiated a long-term educational project that would examine the structural and biochemical changes in glutaredoxin as a function of single-point mutational replacements. Here, we report some of the mutational effects that we have observed to date.

Keywords: glutaredoxin A, educational platform, mutagenesis, protein structure, tertiary, synechocystis sp. PCC 6803

\section{INTRODUCTION}

Glutaredoxin, a member of the glutaredoxin/thioredoxin superfamily, was originally discovered during the search for the electron donor for Escherichia coli ribonucleotide reductase (Holmgren, 1976, 1979). Later, it was determined that glutaredoxins serve not only as the electron donor for a variety of reductant-requiring enzymes, but also as essential components in other cellular processes. For example, glutaredoxins play a critical role in restoring protein function following damage by oxidative stress, as well as mediating the response to heavy metal ion induced damage (Lundstrom-Ljung and Holmgren, 1995). Plant glutaredoxins play strategic roles in signaling pathways, as they participate in the regulation of diverse metabolic pathways. They are also involved in the regulation of gene expression (Couturier et al., 2013). In the case of the glutaredoxin that is the focus of this study, glutaredoxin A from the cyanobacterium Synechocystis sp. PCC 6803 serves as the preferred electron donor for the 2-electron reduction of arsenate to arsenite, catalyzed by arsenate reductase (Li et al., 2003; Lopez-Maury et al., 2003).

As part of the thioredoxin superfamily, glutaredoxin A shares the characteristic thioredoxin fold. This motif is most prominent in prokaryotic glutaredoxins, including glutaredoxin A from Synechocystis sp. PCC 6803, while the thioredoxin fold only exists as a substructure or domain in eukaryotic glutaredoxins (Figure 1) (Eklund et al., 1984). The small size, the ease of purification, and the physiological importance of this protein in both prokaryotes and eukaryotes make Synechocystis sp. PCC 6803 glutaredoxin A an ideal platform on which to base the study of protein mutations.
We previously reported the high-resolution crystal structure of glutaredoxin A from Synechocystis sp. PCC 6803 (Figure 2) (Kim et al., 2012). The structure of the wild-type protein is comprised of the major protein secondary structure elements: four $\alpha$-helical segments, and four mixed parallel: anti-parallel $\beta$-strands that form a single $\beta$-pleated sheet. The assortment of secondary structural elements and the oxidation-reduction activity of glutaredoxin are two characteristics that establish this protein as an ideal template for students to study protein structure/function in detail. The glutaredoxin that we crystallized possesses an $\mathrm{N}$-terminal hexa-His affinity tag to facilitate purification of the protein. The resulting crystal structure is unusual in that the entire N-terminal His-tag extension is completely resolved and each of the histidine residues in the affinity tag is visible in the electron density. The final crystal structure includes two sulfate anions from the crystallization medium. One of the sulfate ions is coordinated at the positive end of the helix dipole of helix 3, while the other sulfate ion is coordinated to the $\mathrm{N}$-terminal hexa-histidine affinity tag. The $\mathrm{pH}$ of the crystallization buffer is 8.0 ; therefore, there is likely very little charge contributed by the histidine side chains that are expected to have $\mathrm{pK}_{\mathrm{a}}$ values near 6.0. The affinity of the negatively charged sulfate ion for this particular aspect of the protein probably results from a combination of the amide backbone charge interactions and shape complementarity of the $\mathrm{N}$-terminus with the sulfate anion.

Another marked difference between the Synechocystis sp. PCC 6803 glutaredoxin A structure and other glutaredoxin structures is the lack of a disulfide bond between the two cysteine 


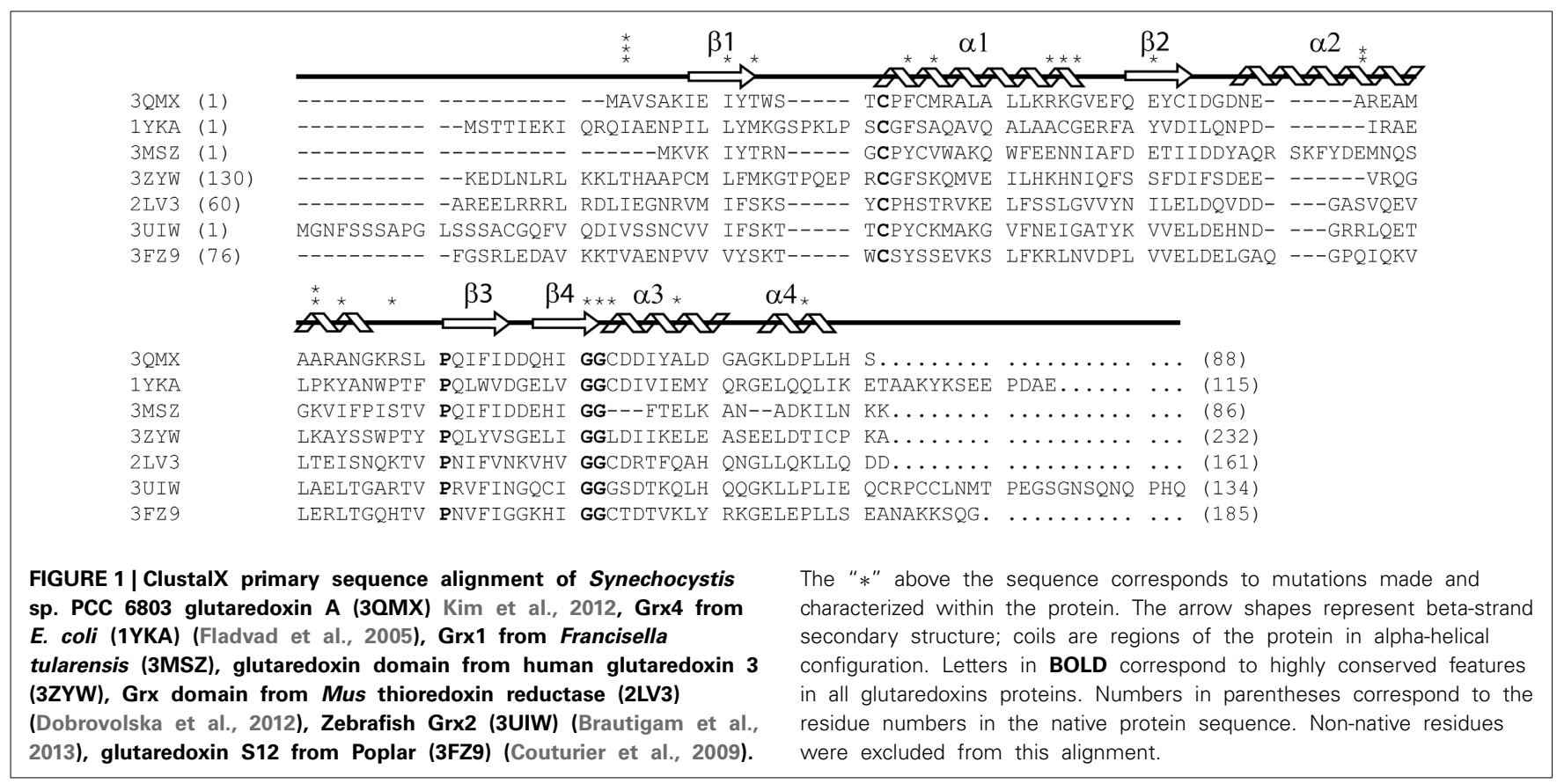

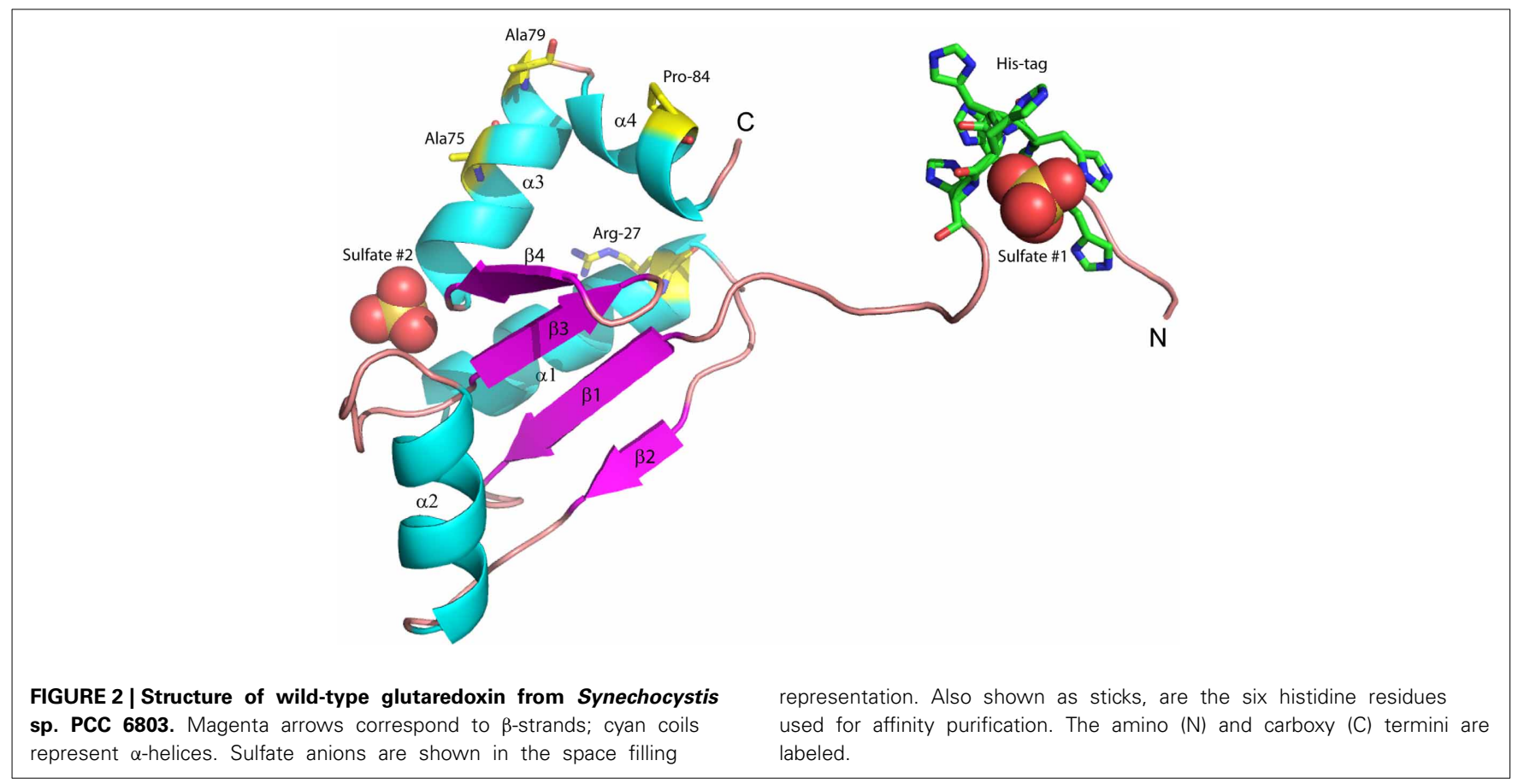

residues at the catalytically-active site, i.e., Cys15 and Cys 18. The oxidation-reduction midpoint potential of the active site disulfide/dithiol couple in Synechocystis glutaredoxin A has been measured at $-225 \mathrm{mV}$ at $\mathrm{pH} 7.0$ (Kim et al., 2012). Typically, glutaredoxins and thioredoxins with redox potentials in this range are found with the active site in the oxidized disulfide form at the conclusion of the purification protocol. However, the X-ray structure of Synechocystis glutaredoxin A unambiguously shows that the active site is fully reduced with no sign of any disulfide formation (Kim et al., 2012). The reasons for this unexpected result are not yet clear.

Site-directed mutagenesis has been used for many years to investigate the detailed function of genes and proteins (Muller et al., 1978). In this technique, the codon of a single amino acid is modified by the introduction of a mutagenic primer in a PCR amplification protocol to produce genes with alternative nucleotide sequences. When translated in a suitable expression system, these mutated genes will produce proteins with an altered 
primary sequence. In most mutagenesis experiments, the selection of the amino acids to be replaced is logically determined. For example, the technique is typically used to focus on specific features of a protein, such as the active sites of enzymes. In contrast, the selection of amino acids for our purposes involves choosing the mutations by random chance, without any preconceived assumption about function or location within the protein. Upon completion of this project, we will have compiled a comprehensive database of biochemical and biophysical information that includes, but is not limited to, the effects of point mutations on protein stability and activity, effects of mutation on solubility, effects of mutation on three-dimensional structure, and effects of mutation on protein crystal formation.

\section{RESULTS AND DISCUSSION}

Widespread mutagenesis studies have previously been carried out with proteins other than glutaredoxins. For example, extensive analysis of the T4 lysozyme protein using a rational selection of sites, uncovered valuable information on the biophysics of protein folding and stability (Baase et al., 2010). However, complete coverage of mutagenesis space has not yet been accomplished for any single protein. This is largely because complete mutagenesis of a protein would require a great deal of labor, and many of the results would be scientifically trivial. In the case of glutaredoxin $\mathrm{A}$, there are 88 amino acids in the complete protein, making it one of the smallest redox proteins known. If each site were mutated to one of the other 19 amino acids, 1672 new genes will have to be prepared. To accomplish this herculean task, we have integrated the research component of this project into an educational framework as an adjunct to a traditional biochemistry or protein-engineering course. A further advantage arises from the fact that the task can be accelerated by involving students from many different schools who are linked by a common database from a centralized campus via the Internet. A pilot version of this collaborative project has already collected information on protein solubility, crystallization, and structure. We have completed 26 random mutations in the three consecutive semesters that we have offered this course. Four new crystal structures have been generated.

\section{EFFECTS OF RANDOMLY SELECTED MUTATIONS}

Given that our mutations are randomly selected, at least four possible outcomes are expected. First, the mutated gene yields a protein that is unable to fold, and thus, would exhibit very low solubility. In this case, the protein could be sequestered in inclusion bodies in the bacteria, it could be completely proteolyzed, or it would yield only extremely low amounts of soluble protein. Second, the protein may be soluble, but is unable to crystallize under our current crystallization conditions. Third, the protein may crystallize, but the resulting crystals are too small for rapid analysis. Fourth, large, single-protein crystals are formed, and are amenable for crystallographic analysis.

We assume that many of the randomly selected mutations in the glutaredoxin gene will result in protein misfolding. For example, mutations within the hydrophobic core of most proteins are not well tolerated (Guo et al., 2004). Of the 27 mutations that we have characterized, three-T11Y, L25S, and G68Y-have resulted in the formation of an insoluble protein. The Gly68Gly69 sequence is highly conserved within the glutaredoxin family (Figure 1). This di-glycine motif makes a bend in a loop between $\beta$-strand 4 and $\alpha$-helix 3 ; hence, this sequence is likely to be an essential determinate of the native structure. The introduction of bulky tyrosine residues may impose considerable steric hindrance on this loop, thus, resulting in misfolding and this may explain the insolubility of the G68Y variant. In contrast, the G69F mutation is soluble, but did not crystallize. The T11Y mutation occurs at the apex of $\beta$-strand 2 , adjacent to critical cysteine residues in the molecule. The introduction of a bulky side chain at this point may destabilize the core packing at this critical position.

\section{MUTANTS OF GLUTAREDOXIN A THAT PRODUCE PROTEIN CRYSTALS}

Four of our mutant proteins produced crystals suitable for X-ray structure determination (Tables 1, 2). At least 10 of the 28 mutations that we have examined have produced crystalline material (Table 1). We define "crystalline material" as protein crystals that form either thin, fibrous crystals or protein crystals that are too small to justify further effort. Proteins that form fibrous crystals typically add protein molecules to the crystal lattice preferentially along one growth axis. In our case, A2I, A2T, I9L, R27V, K28C, A43I, A43T, A49H, A49W, and R55A variants each produced sub-optimal protein crystals. Interestingly, both hydrophobic and polar variants replacing Ala2 (A2I, A2T, and A2Y) produced soluble protein, but only A2I and A2T grew protein crystals, albeit crystals of poor quality. This particular locus on the molecule is in a flexible loop at the beginning of the polypeptide chain and is not involved in any direct crystal contacts; however, there are residues from symmetry-related molecules within $5 \AA$ of this locus. The introduction of a bulky mutation such as Tyr could add as many as 6 new amino acid neighbors within a $5 \AA$ sphere that could interfere with crystal packing (Table 1).

The I9L replacement should be a conservative change in the protein, as the physio-chemical properties and side chain volumes of isoleucine and leucine are very similar. Mutational studies of other proteins have concluded that these two amino acids are largely interchangeable and that replacement of one by the other generally has little or no effect on a protein's biophysical properties (Betts and Russell, 2003). Accordingly, one would predict that such a minor change should be tolerated by crystal packing. However, this mutation did affect crystal formation. The reason for this change is still unclear. $\mathrm{K} 28 \mathrm{C}$ is another interesting mutation. In wild-type glutaredoxin, Lys 28 is involved in a complex salt-bridge between $\alpha$-helices 1, 2, and 3. One would expect that the substitution of an essential, conserved residue like Lys 28 with a slightly polar side chain like Cys, should interfere with the biophysical properties of the molecule. However, this mutation yields soluble protein and small protein crystals.

\section{FOUR NEW MUTANT STRUCTURES}

Our random mutagenesis approach has yielded four new crystal structures of glutaredoxin A, one for each of the following variants: R27L, A75I, A79S, and P84R (Table 2). Each of these crystal structures provides information about the structure and 
Table 1 | List of currently studied mutations in Synechocystis sp. PCC 6803 glutaredoxin A.

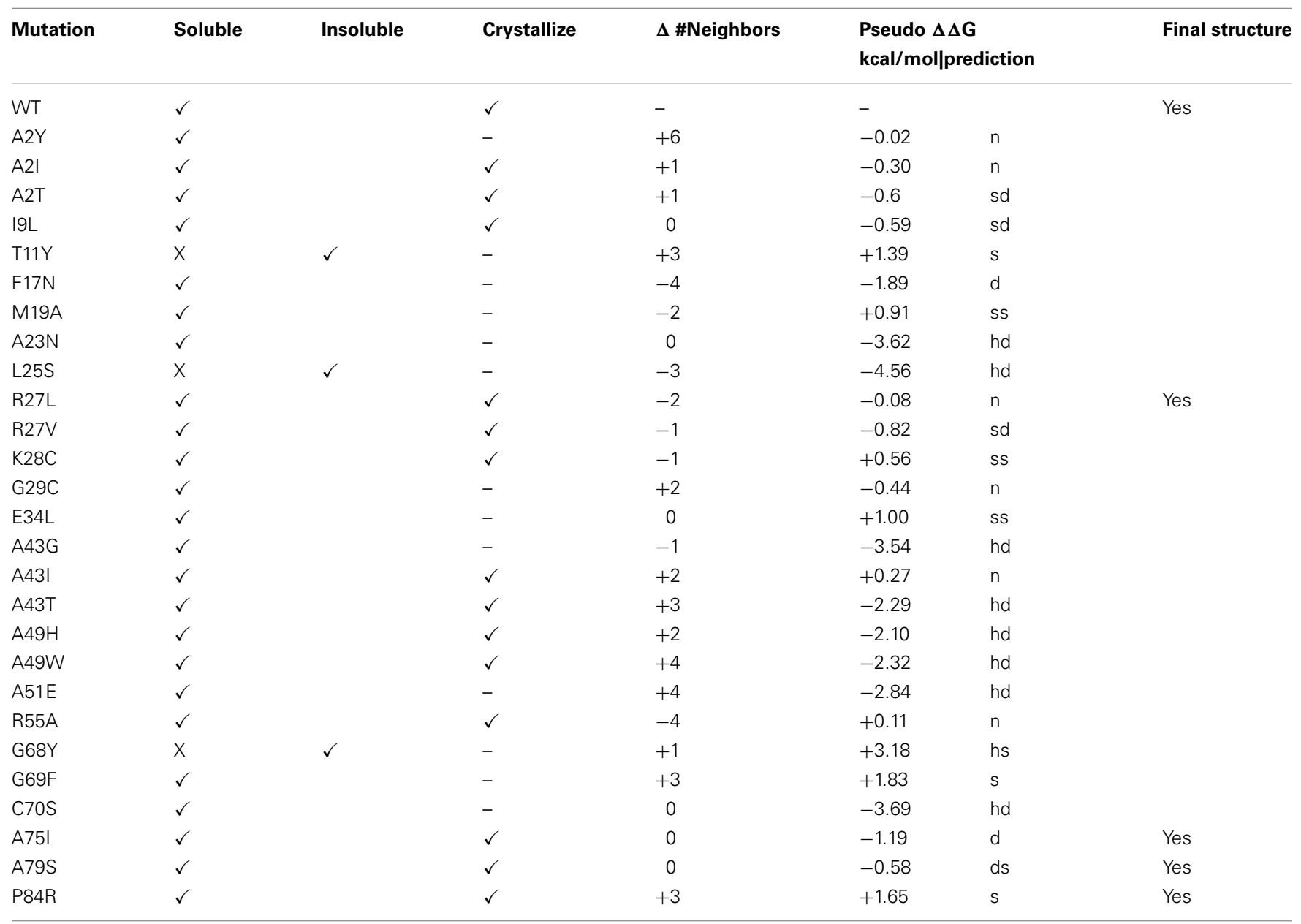

Soluble $=$ mutant protein could be purified from bacterial lysate.

Insoluble = no mutant protein could be purified from bacterial lysate.

Crystallize = were crystals of any quality grown?

$\Delta \#$ Neighbors $=$ residues which could cause potential collisions in this crystallographic setting

Pseudo $\Delta \Delta G$ (kcal/mol) n, neutral; sd, slightly destabilizing; s, stabilizing; d, destabilizing; hd, highly destabilizing; ss, slightly stabilizing; hs, highly stabilizing.

Structure $=$ Was a refined crystal structure produced?

function of the glutaredoxin protein, in addition to information about protein crystal formation and protein structure in general.

The overall structure of the $\mathrm{R} 27 \mathrm{~L}$ variant is virtually indistinguishable from that of the wild-type protein. The RMSD (root-mean-square deviation) between both structures is 0.099 $\AA$ over all atoms, and the Leu27 substitution was confirmed in the refined experimental electron density (Figure 3). Arg27 occurs at the C-terminal end of $\alpha$-helix 1 of the wild-type protein and potentially forms a $\mathrm{H}$-bond with Tyr74. In addition, Arg27 occurs in a cluster of other basic residues (...K-R-K...), so it is likely that it is part of a localized positively charged patch on the surface of the protein. The leucine substitution would obviously disrupt this patch, but little else would be predicted to occur. Nevertheless, the diffraction resolution of the crystals of the R27L variant was significantly increased compared to the data obtained with wild-type glutaredoxin A. Diffraction resolution is an optical property of crystals, which is dictated by the inherent order of a crystal lattice to diffract X-rays. The lower the value for the resolution, the better the crystal is ordered and the better it can provide X-ray diffraction data that can resolve the measured distance between atoms. Crystals of the wild-type protein diffract to $1.7 \AA$ resolution, while the $\mathrm{R} 27 \mathrm{~L}$ mutation produced crystals that diffract X-rays to $1.2 \AA$ resolution. The sizes of the crystals of the wild-type and R27L variants were similar, so the increase in diffraction cannot be explained by larger crystals. Furthermore, as all data were collected at the same X-ray source, increased X-ray intensity cannot explain this increase in resolution. Therefore, it is likely that a property of the mutation contributed to this effect. There is a possibility that more efficient hydrophobic packing between symmetry-related molecules, mediated by the leucine replacement, contributed to more efficient crystal packing. This observation is essentially what has been described as surface entropy reduction (SER). SER is an in silico technique that predicts mutants, which could possibly facilitate 
Table 2 | Data collection and refinement statistics.

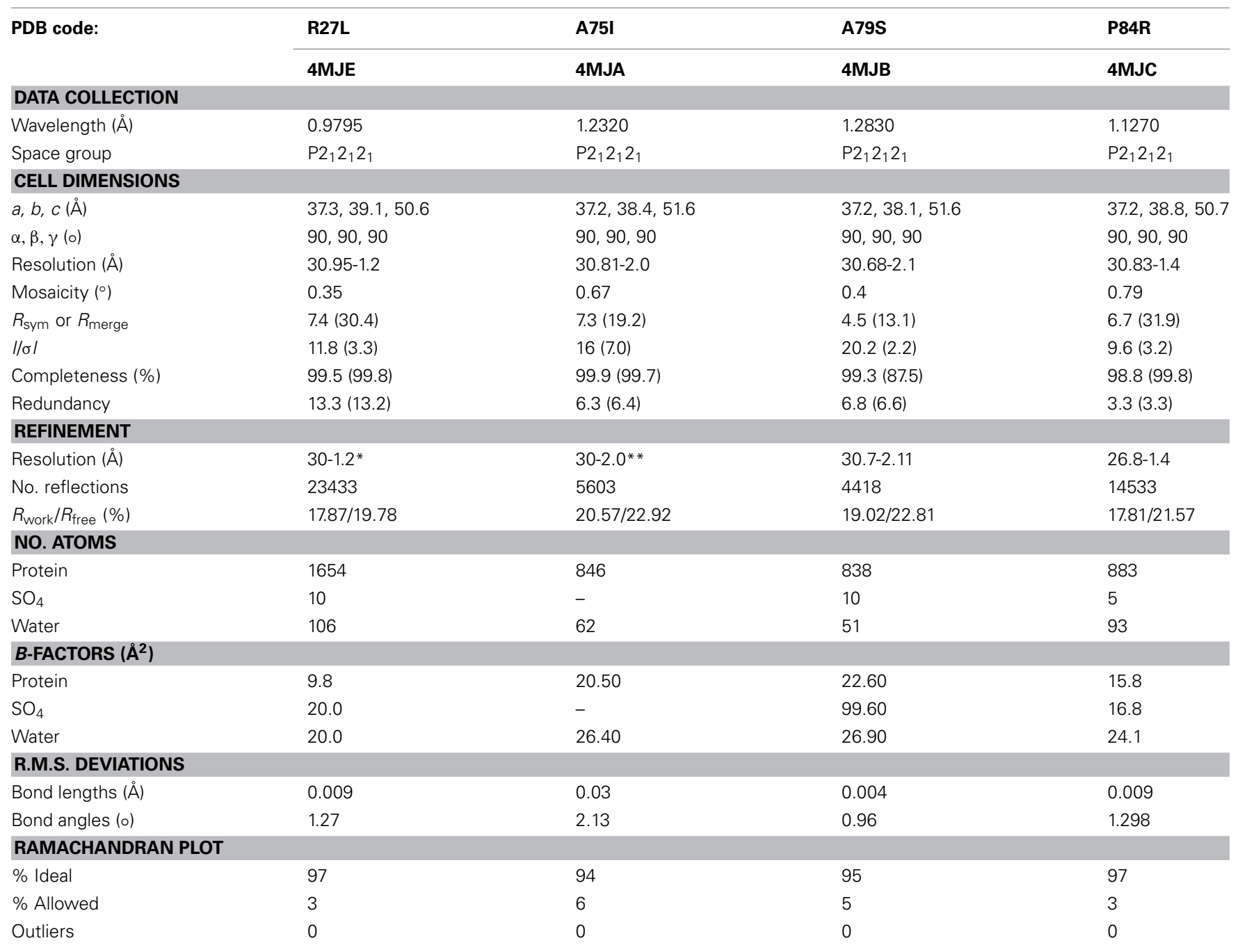

${ }^{*}$ Coordinates refined with "riding" hydrogen atoms.

${ }^{* *}$ No sulfate ions were present in this crystal structure.

more productive contacts among protein molecules in a crystal lattice (Goldschmidt et al., 2007). More efficient crystal packing would then result in higher X-ray scattering angles and higher overall resolution. Such a result, if it were to turn out to be a general effect, could provide information that would allow the engineering of better diffracting protein crystals. Potentially, if a low-resolution structure revealed critical crystal contacts, then mutations could be introduced to optimize those crystal contacts and increase overall diffraction without compromising the structure or activity of the protein.

Due to the location of Ala75, one might have predicted that changes in structure and function observed for the A75I and the A79S variants would be subtle. These replacements are located at the extreme C-terminus of the protein in $\alpha$-helix 4 (Figure 2), and both are exposed to the outside surface of the helix. Neither is involved in crystal packing interactions nor core packing interactions, and therefore, these positions are predicted to be highly tolerant of modification. However, in the case of the A79S mutant,
Table 3 | Changes in helix properties in helix $\alpha-4$ of glutaredoxin (Bansal et al., 2000; Kumar and Bansal, 2012).

\begin{tabular}{lllll}
\hline Mutation & Twist $\left({ }^{\circ}\right)$ & $n$ & height $(\AA)$ & Bending angle $\left(^{\circ}\right)$ \\
\hline Wild-type & 102.2 & 3.52 & 1.67 & 7.3 \\
R27L & 101.1 & 3.56 & 1.76 & 7.8 \\
A75I & 101.1 & 3.60 & 1.65 & 7.6 \\
A79S & 101.3 & 3.55 & 1.68 & 5.4 \\
P84R & 101.8 & 3.54 & 1.66 & 11.8 \\
\hline
\end{tabular}

$n=$ amino acids/turn.

$h(\AA)=$ unit height of the helix.

Bending angle $\left({ }^{\circ}\right)=$ Bending angle between successive local helix axes.

there are minor structural changes observed in the geometry of $\alpha$-helix 4 . There is a measurable decrease in the twist of the helix, a decrease in the helix-bending angle of $\alpha$-helix 4 (Table 3 ). The significance of these minor changes remains to be explored. 

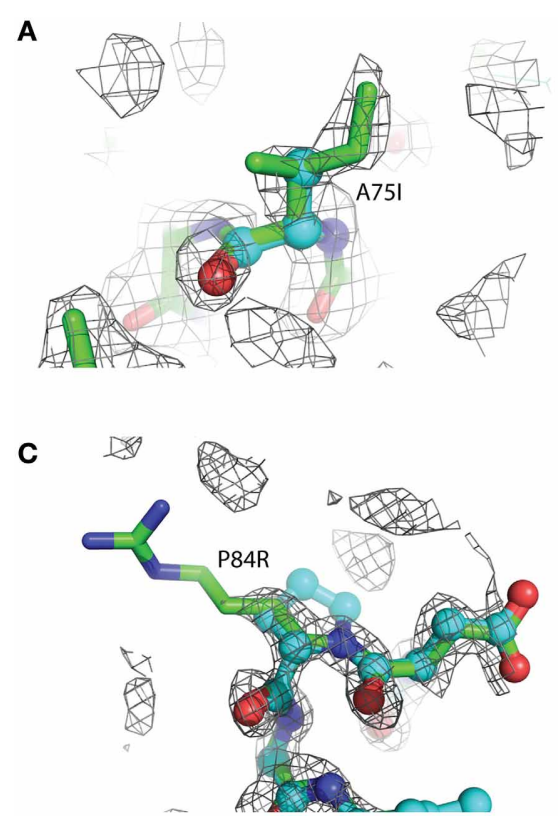

FIGURE 3 | Representative 2Fo-Fc electron density maps, contored at the $1 \sigma$ level, of each of the four mutations described. (A) A75I: The wild-type Ala is shown as light blue ball-and-stick, while the mutant lle is shown as green sticks. The chicken wire shape around the amino acid represents the electron density carved from the final refined structure. (B) A79S: Ala79 is shown as blue balls-and-sticks. The mutant Ser is shown as green sticks. In this case, two conformations of Ser79 can be modeled. Both rotamers were

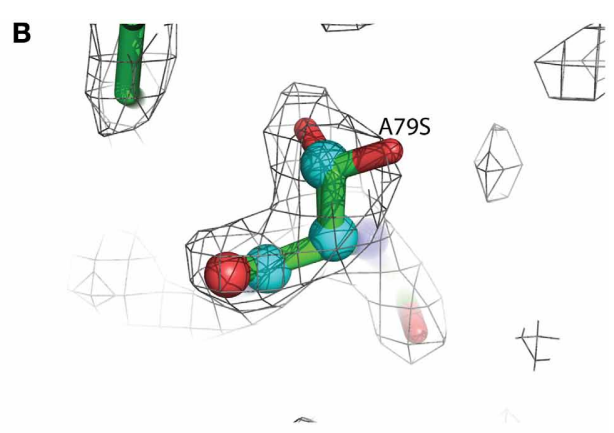

D

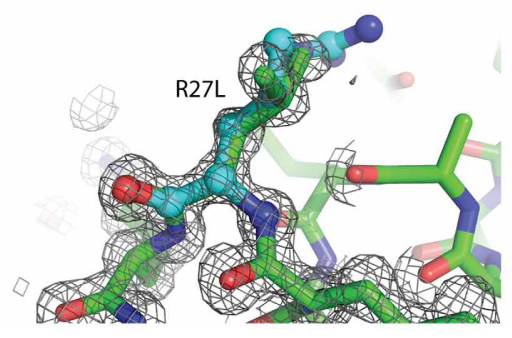

used at 50\% occupancy in the refinement of this structure. (C) P84R: wild-type Pro 84 is shown as blue balls-and-sticks. Mutant Arg79 is shown as green sticks. It is common for the more flexible amino acids, such as Arg or Lys, to exhibit abbreviated electron density due to the rotary motion of the side chain. (D) R27L: The Arg27 present in the wild-type protein is shown as blue balls-and-sticks. The structure of the leucine replacement at this position has been superimposed on top of the wild-type and is shown as green sticks.
The P84R mutation is interesting from a structural perspective. Firstly, Pro84 occurs at the beginning of an $\alpha$-helix that is bent by almost $90^{\circ}$ relative to a neighboring $\alpha$-helix in the structure. The only impetus for this dramatic bend is the maintenance of a protected hydrophobic core. A proline is present at this locus in other glutaredoxin molecules, but not all. A Blast search using the Synechocystis sp. PCC 6803 glutaredoxin A sequence revealed that $48 \%$ of 98 glutaredoxin sequences utilize proline at a homologous position in the primary sequence (data not shown). As proline does not provide a free backbone amide hydrogen to form an $\mathrm{H}$-bond to the $i+4$ backbone carbonyl, it rarely occurs within a helix (Kim and Kang, 1999). In the wild-type glutaredoxin structure, there is only one $i, i+4$ backbone $\mathrm{H}$-bond interaction to stabilize $\alpha$-helix 4 (Figure 2). While mutation of Pro84 to arginine does not restore the complete backbone H-bond pattern typical of $\alpha$-helices, it does appear to alleviate strain on the peptide backbone so an additional $i, i+4$ backbone $\mathrm{H}$-bond interaction can form between the backbone carbonyl of Asp83 and the backbone amine of Ser88(Figure 4).

\section{CHANGES IN PROTEIN STABILITY}

Many of the mutations that we introduce using our random selection method could compromise protein stability in many different ways. For example, secondary structure may be disrupted by introducing a residue incompatible with $\beta$-sheet formation. The resulting misfolded protein could result in an insoluble protein in E. coli. To predict changes in stability, we submitted our point mutants to the Site Directed Mutator (SDM) server for analysis (Table 1). This procedure returns a prediction on whether the point mutation is neutral, stabilizing, or destabilizing based on environment-specific substitution tables and the resulting change in free energy (Worth et al., 2011). Large negative $\Delta \Delta G$ correlate with varying degrees of instability, while large positive $\Delta \Delta G$ values correlate with stabilizing energies. In general, the glutaredoxin A mutations that yielded soluble protein were predicted to be either neutral, stabilizing or destabilizing, but the range in free energies was small. The mutants at the extremes of the free energy distribution tended to yield insoluble protein. The exception to this is the C90S mutant, where the calculated free energy difference was negative $(-3.69 \mathrm{kcal} / \mathrm{mol})$, and therefore, predicted to be highly destabilizing (hd), yet the purified protein is soluble. The energetics of point mutations is clearly a complex problem; however, with more mutations, it is possible that the predictive abilities of the free energy algorithms will correlate more closely with our observations.

\section{INCONSISTENCIES WITH THE ORTHORHOMBIC CRYSTAL LATTICE}

Ideally, we would like to screen each soluble, purified glutaredoxin A mutation for its own unique crystallization condition, but we are limited in resources and by the time our students can spend with this research project. Therefore, we have concentrated on a single crystallographic condition. This restriction obviously imposes limitations on our method, but it also addresses the 


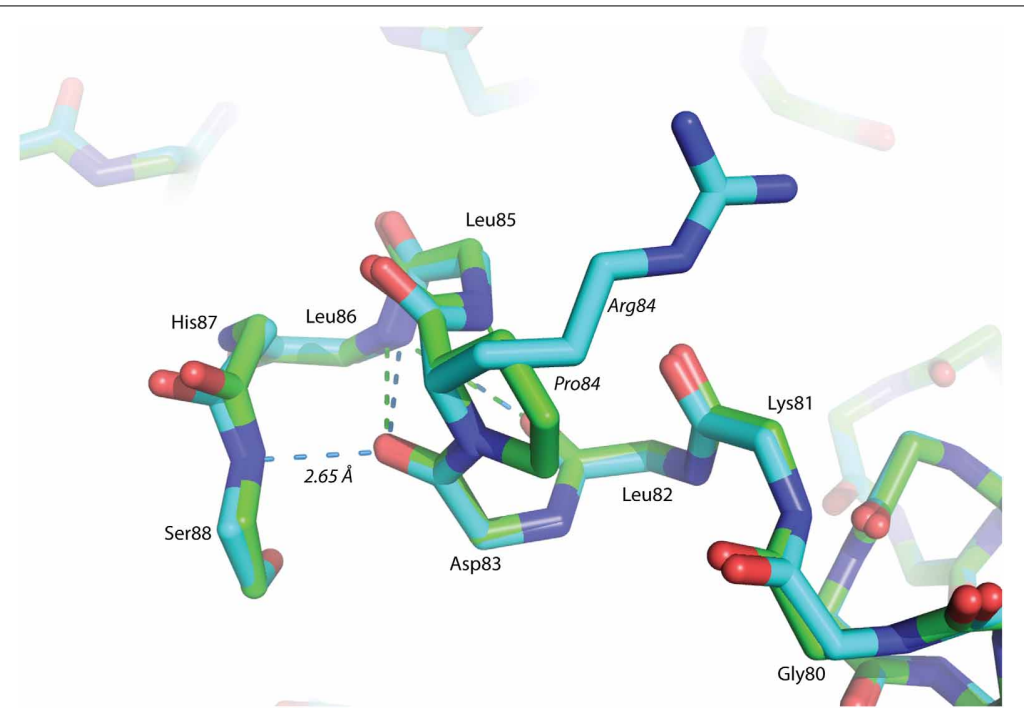

FIGURE 4 | Superposition of helix $\alpha-\mathbf{4}$ from Glutaredoxin A. The green structure is wild-type, while the blue structure is mutant P84R. The dashed-lines are backbone $\mathrm{H}$-bonds that stabilize $\alpha$-helix 4. One additional consensus $\mathrm{H}$-bond has been included in the P84R structure.

question about how variable or plastic a protein can be yet pack into a given lattice. Most of our mutants form suboptimal protein crystals. It is possible that the modifications we make to the glutaredoxin protein produces changes that cannot form productive crystal contacts, and therefore, disfavors efficient crystal packing. To assess this, we studied the change in the number of neighboring inter-molecular residues (within $5 \AA$ ) between mutant and wild-type glutaredoxin, assuming orthorhombic crystal packing (Table 1). More inter-molecular neighbors would likely correlate with potential clashes; whereas, fewer contacts could correlate with missing interactions. One would predict that extremes in both would make the protein less likely to crystallize in the orthorhombic setting we have measured in the wild-type glutaredoxin. Interestingly, in the case of the mutations that produced crystal structures, there is no obvious correlation between either adding or subtracting inter-molecular neighbors. The R27L crystal structure lacks two neighbors; the A75I and A79S mutant have no net differences, while the P84R mutant adds three possible interactions (Table 1). This result could reflect the plastic nature of proteins that allows them to adopt new shapes. As we have also observed that most of our soluble glutaredoxin mutants can form crystalline material, it is therefore, possible that changing the number of clashes either could make nucleation more probable or alternatively, could hinder crystal growth. As our dataset increases in size with time, we will be able to make more definitive conclusions.

\section{SULFATE BINDING}

Since ammonium sulfate is used in the crystallization medium of these glutaredoxin A crystals, there are two sulfate anions coordinated to the wild-type and to the mutated glutaredoxin A structures. Interestingly, the A75I mutation does not coordinate sulfate ions in its crystal structure. In the wild-type structure, sulfate \#2 (Figure 2) is $10.6 \AA$ from the $\mathrm{C} \alpha$ atom of Ile75, while sulfate \#1 (Figure 2) is $35.2 \AA$ distant. The large distance between the site of the mutation and the ligand binding site discounts any direct effect on sulfate binding. A cogent rationale for the absence of sulfate anions in the electron density is still unknown; however, we can draw conclusions about the effects of sulfate binding to the A75I crystal structure. As there is no sulfate anion bound to the N-terminus, the His-tag of A75I is considerably more disordered that the other glutaredoxin structures that do coordinate sulfate. The temperature factor is a measure of thermal motion within a crystal structure; atoms with higher temperature factors are thought to be more disordered. The average refined temperature-factors for the artificial N-terminus of wild-type glutaredoxin A (the loop including the His-tag residues) including the coordinated sulfate anion is $17.7 \AA^{2}$. The same residues in the A75I structure without a coordinated sulfate anion show an average temperature factor of $32.6 \AA^{2}$ (Figure 5). While the elevated temperature factors can be explained by the lack of sulfate coordination, the rationale for the lack of this anion in this mutation is still unknown.

\section{CONCLUSION}

Our random point mutant selection scheme will ultimately cover all of the 1672 possible mutations of Synechocystis sp. PCC 6803 glutaredoxin A, while simultaneously teaching students about biotechnology and protein structure. There are, however, several weaknesses to this method. Due to time constraints, we have to restrict our crystallization experiments to one specific condition; one that had originally been identified during crystallization trials for the wild-type protein. While we will clearly learn how mutation affects crystal growth under these conditions, a broader-ranging, independent approach would be more informative. Another weakness of this project is that we seek to involve students from different institutions with a wide range of backgrounds and training. As such, it introduces considerable variability in the skill 


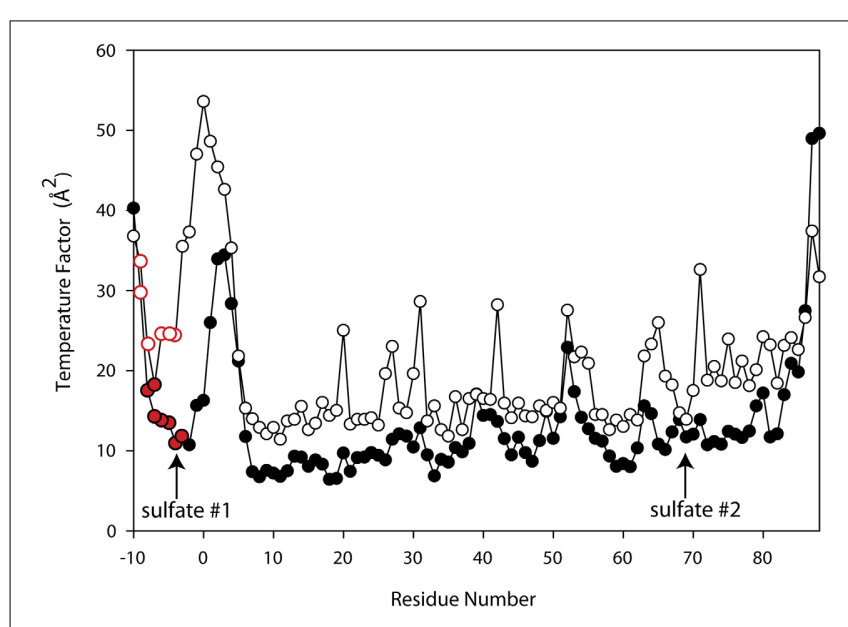

FIGURE 5 | Superimposed Temperature Factor plot of wild-type glutaredoxin (solid circles) (3QMX) vs. A75I (white circles). The red color depicts the His-residues that make up the His-tag of the molecule. The arrows show the location of the shortest sulfate:amino acid distance.

with which different amino acid replacements will be carried out and analyzed. While only the most diligent students will be able to make significant headway in this project, the rewards of involving a large number of students and potentially exciting them about science surely outweighs the negative aspects.

It is hoped that we will ultimately compile an extensive database that will include information to stabilize protein folds or, perhaps, to reveal techniques that improve protein crystal diffraction. However, in the limited coverage of mutational space that we have already explored, it is clear that not all mutations can be immediately understood, and their effects are still reasonably unpredictable. This is one of the primary concepts that we try to instill in students from the beginning.

\section{MATERIALS AND METHODS HETEROLOGOUS EXPRESSION}

The mutagenesis protocol that we use is described in detail (Kim et al., 2012). A full description of the plasmid is described in Kim et al. (2012).

$1.0 \mu \mathrm{g}$ of plasmid DNA was added to $100 \mu \mathrm{l}$ of chemically competent BL-21(DE3) E. coli in a $1.5 \mathrm{ml}$ microfuge tube. This mixture was incubated on ice for $30 \mathrm{~min}$. The cells were then heat-shocked at $37^{\circ} \mathrm{C}$ in a water-bath for $45 \mathrm{~s} .650 \mu \mathrm{l}$ of SOC medium were added to each microfuge tube. The plasmid DNA/BL-21(DE3) mixture and the SOC medium were placed in a $37^{\circ} \mathrm{C}$ incubator, shaken at 225 r.p.m. for a $1 \mathrm{~h}$ and subsequently plated onto an agar substrate containing $100 \mu \mathrm{g} \mathrm{ml}$ 87,221 ampicillin. The plates were then placed in a stationary incubator at $37^{\circ} \mathrm{C}$ for $12 \mathrm{~h}$. Start-up cultures were prepared by inoculating individual colonies from the plasmid transformation plate into $25 \mathrm{~mL}$ of Luria-Bertani medium (LB) and $100 \mu \mathrm{g}$ $\mathrm{ml}^{-1}$ of ampicillin. This culture was incubated at $37^{\circ} \mathrm{C}$ while shaking at 225 r.p.m. overnight. The start-up LB cultures were added to $1 \mathrm{~L}$ Terrific broth (TB) to maximize bacterial cell yield. TB cultures were incubated at $37^{\circ} \mathrm{C}$, with shaking at 225 r.p.m. until OD600 reached 1.3, at which point $400 \mu$ l of isopropyl $\beta$-D-1-thiogalactopyranoside (IPTG) was introduced to induce protein expression. Prior to IPTG induction, a $500 \mu \mathrm{l}$ sample of un-induced cells was set aside for PAGE electrophoresis. TB cultures were incubated for a $12 \mathrm{~h}$ period at $20^{\circ} \mathrm{C}$ for a $3-4 \mathrm{~h}$ period at $37^{\circ} \mathrm{C}$, with shaking at 225 r.p.m. and subsequently spun down at 7200 r.p.m. in a centrifuge at $4^{\circ} \mathrm{C}$. A post-induction gel electrophoresis sample was taken prior to centrifugation to confirm heterologous expression. Cell pellets were collected, flash-frozen in liquid nitrogen, and stored at $-80^{\circ} \mathrm{C}$ until lysis.

\section{PURIFICATION}

Following SDS-PAGE verification of heterologous protein induction in bacteria, cell cultures were thawed at $0^{\circ} \mathrm{C}$, suspended in a lysis buffer $(30 \mathrm{mM}$ Tris buffer, $\mathrm{pH} 8.0$, containing $500 \mathrm{mM}$ $\mathrm{NaCl}$ ), vortexed, and then lysed at 20,000 psi in a Microfluidics $\mathrm{M}-100 \mathrm{EH}$ microfluidizer. Lysed cells were centrifuged at 42,000 r.p.m. for $45 \mathrm{~min}$ at $4^{\circ} \mathrm{C}$; the supernatant was subsequently collected and bound to Ni-NTA resin for $12 \mathrm{~h}$. Glutaredoxin-bound Ni-NTA resin was washed with $30 \mathrm{mM}$ Tris buffer, $\mathrm{pH} 8.0$, containing $500 \mathrm{mM} \mathrm{NaCl}$ plus $30 \mathrm{mM}$ imidazole until the $\mathrm{OD}_{280}$ of the eluent was $\leq 0.010$. Glutaredoxin A was eluted from the column with $40-50 \mathrm{ml}$ of $30 \mathrm{mM}$ Tris buffer, $\mathrm{pH} 8.0$, containing $300 \mathrm{mM}$ imidazole, plus $500 \mathrm{mM} \mathrm{NaCl}$. Post-elution protein purity was confirmed by SDS-PAGE using a Phastgel apparatus (GE Healthcare). Protein concentrations were determined either using the BioRad Protein Assay with bovine serum albumin as a standard (Bradford, 1976), or by using reference parameters calculated from the molecular weight and calculated extinction coefficient of the mutant in question (Gill and Von Hippel, 1989). Glutaredoxin A mutants were concentrated to approximately $6 \mathrm{mg} / \mathrm{ml}$ and further purified by gel filtration on a Superdex 75 column $(5 \times 300 \mathrm{~mm})$. Protein was re-concentrated for crystallization using a $10 \mathrm{~K}$ cutoff Amicon spin concentrator.

\section{CRYSTALLIZATION}

Crystallization trials of all mutant glutaredoxin proteins utilized the hanging-drop vapor-diffusion method. Noting previously identified glutaredoxin A wild-type crystallization conditions, 24 well trays were set up with a horizontally varying salt gradient (1.1-1.5 M ammonium sulfate), and a vertically varying $\mathrm{pH}$ gradient ( $\mathrm{pH}$ 7.0-8.2 HEPES buffer). 1\% PEG400 (w/v) was included throughout. Crystal droplets consisting of $2 \mu \mathrm{l}$ protein solution and $2 \mu \mathrm{l}$ reservoir solution were added and trays were placed in $23^{\circ} \mathrm{C}$ for crystallization. Long, rod-like structures displaying the typical morphology of glutaredoxin crystals generally appeared after $24 \mathrm{~h}$; however, some mutants grew crystals in as much as 2 weeks.

\section{STRUCTURE SOLUTION}

The crystals were captured on nylon loops and flash frozen in liquid $\mathrm{N}_{2}$. Initial data sets were collected on a Rigaku ScreenMachine. Subsequent data sets were collected at SLAC beamline 7-1. The data were collected at $90 \mathrm{~K}$. X-ray data was processed with imosflm (Battye et al., 2011) and the data were scaled using SCALA as a part of the CCP4 package (Winn et al., 
2011). $10 \%$ of the data were allocated for R-free cross validation. A summary of the crystal statistics are presented in Table 2.

Structures of all mutants were solved by molecular replacement using 3QMX as the target structure. We used the Phaser module as implemented in the Phenix package for this calculation. Subsequent electron density for the protein was manually fit using Coot (Emsley et al., 2010). Electron density assigned as water molecules was automatically assigned by Phenix during the final stages of refinement. Each water molecule was checked manually in Coot, and in cases that were missed by Phenix, waters were added manually to density that met the H-bonding geometry and inter-atomic distance criteria established by Phenix. Structural refinement was also performed using the Phenix package (Adams et al., 2010).

\section{MUTANT ANALYSIS}

The thermodynamic stability for each single site mutant in our data set was predicted using the SDM web server (Worth et al., 2011). This website calculates changes in stability that likely result from single site mutations in proteins. Factors such as the physiochemical differences between the wild-type and mutant protein within the known protein structure are used to compute a free energy term (Worth et al., 2011). A significant change in effective free energy correlates with either with a stabilizing or a destabilizing prediction (Table 1).

Potential conflicts with the wild-type crystal packing were analyzed in Pymol (Schrodinger, 2013). First, the wild-type residue of interest was selected in Pymol. Then, complete symmetry-related molecules in crystal lattice were expanded in a sphere $5 \AA$ around that amino acid. All of the residues within $5 \AA$ of the amino acid were selected and counted. Simulated mutants were computed using the Pymol mutagenesis wizard. The most common rotamer was selected without any subsequent energy minimization. The same symmetry expansion and residue selection was performed as was described from the wild-type protein. The net difference between the number of neighboring residues in the wild-type protein and the mutant protein was reported as $\Delta$ \# Neighbors (Table 1).

\section{ACKNOWLEDGMENTS}

This work was supported by a grant from the $\mathrm{CH}$ Foundation (to Roger B. Sutton) and the U.S. Department of Energy (DE-FG03-99ER20346 to David B. Knaff). These two funding sources contributed equally to supporting the project. Portions of this research were carried out at the Stanford Synchrotron Radiation Lightsource, a Directorate of SLAC National Accelerator Laboratory and an Office of Science User Facility operated for the U.S. Department of Energy Office of Science by Stanford University. The SSRL Structural Molecular Biology Program is supported by the DOE Office of Biological and Environmental Research, and by the National Institutes of Health, National Institute of General Medical Sciences (including P41 GM103393) and the National Center for Research Resources (P41 RR001209). The contents of this publication are solely the responsibility of the authors and do not necessarily represent the official views of NIGMS, NCRR or NIH. The authors would like to acknowledge the assistance of Dr.
Jatindra N. Tripathy (Center for Biotechnology and Genomics, Texas Tech University) in helping to supervise the course (BTEC 5338) at Texas Tech University where the mutational study was initiated. In addition, the authors would like to thank students from Wayland Baptist University, Eastern New Mexico University, Southern Illinois University Edwardsville, The Air Force Academy, and Baylor University for contributing. All structures and structure factors have been deposited with the PDB under the following accession codes: wild-type glutaredoxin (3QMX), glutaredoxin A-A75I (4MJA), glutaredoxin A-A79S (4MJB), glutaredoxin A-P84R (4MJC), glutaredoxin A-R27L (4MJE).

\section{REFERENCES}

Adams, P. D., Afonine, P. V., Bunkoczi, G., Chen, V. B., Davis, I. W., Echols, N., et al. (2010). PHENIX: a comprehensive Python-based system for macromolecular structure solution. Acta Crystallogr. D Biol. Crystallogr. 66, 213-221. doi: 10.1107/S090744490 9052925

Baase, W. A., Liu, L., Tronrud, D. E., and Matthews, B. W. (2010). Lessons from the lysozyme of phage T4. Protein Sci. 19, 631-641. doi: 10.1002/pro.344

Bansal, M., Kumar, S., and Velavan, R. (2000). HELANAL: a program to characterize helix geometry in proteins. J. Biomol. Struct. Dyn. 17, 811-819. doi: 10.1080/07391102.2000.105 06570

Battye, T. G., Kontogiannis, L., Johnson, O., Powell, H. R., and Leslie, A. G. (2011). iMOSFLM: a new graphical interface for diffraction-image processing with MOSFLM. Acta Crystallogr. D Biol. Crystallogr. 67, 271-281. doi: 10.1107/S0907444910048675

Betts, M. J., and Russell, R. B. (2003). "Amino acid properties and consequences of substitutions," in Bioinformatics for Geneticists, eds I. C. Gray and M. R. Barnes (Southern Gate Chichester, UK: John Wiley \& Sons, Ltd.), 289-314.

Bradford, M. M. (1976). A rapid and sensitive method for the quantitation of microgram quantities of protein utilizing the principle of protein-dye binding. Anal. Biochem. 72, 248-254.

Brautigam, L., Johansson, C., Kubsch, B., McDonough, M. A., Bill, E., Holmgren, A., et al. (2013). An unusual mode of iron-sulfur-cluster coordination in a teleost glutaredoxin. Biochem. Biophys. Res. Commun. 436, 491-496. doi: 10.1016/j.bbrc.2013.05.132

Couturier, J., Chibani, K., Jacquot, J. P., and Rouhier, N. (2013). Cysteinebased redox regulation and signaling in plants. Front. Plant Sci. 4:105. doi: 10.3389/fpls.2013.00105

Couturier, J., Koh, C. S., Zaffagnini, M., Winger, A. M., Gualberto, J. M., Corbier, C., et al. (2009). Structure-function relationship of the chloroplastic glutaredoxin S12 with an atypical WCSYS active site. J. Biol. Chem. 284, 9299-9310. doi: 10.1074/jbc.M807998200

Dobrovolska, O., Shumilina, E., Gladyshev, V. N., and Dikiy, A. (2012). Structural analysis of glutaredoxin domain of Mus musculus thioredoxin glutathione reductase. PLoS ONE 7:e52914. doi: 10.1371/journal.pone.0052914

Eklund, H., Cambillau, C., Sjoberg, B. M., Holmgren, A., Jornvall, H., Hoog, J. O., et al. (1984). Conformational and functional similarities between glutaredoxin and thioredoxins. EMBO J. 3, 1443-1449.

Emsley, P., Lohkamp, B., Scott, W. G., and Cowtan, K. (2010). Features and development of Coot. Acta Crystallogr. D Biol. Crystallogr. 66, 486-501. doi: 10.1107/S0907444910007493

Fladvad, M., Bellanda, M., Fernandes, A. P., Mammi, S., Vlamis-Gardikas, A., Holmgren, A., et al. (2005). Molecular mapping of functionalities in the solution structure of reduced Grx4, a monothiol glutaredoxin from Escherichia coli. J. Biol. Chem. 280, 24553-24561. doi: 10.1074/jbc.M500679200

Gill, S. C., and Von Hippel, P. H. (1989). Calculation of protein extinction coefficients from amino acid sequence data. Anal. Biochem. 182, 319-326. doi: 10.1016/0003-2697(89)90602-7

Goldschmidt, L., Cooper, D. R., Derewenda, Z. S., and Eisenberg, D. (2007). Toward rational protein crystallization: a Web server for the design of crystallizable protein variants. Protein Sci. 16, 1569-1576. doi: 10.1110/ps. 072914007 
Guo, H. H., Choe, J., and Loeb, L. A. (2004). Protein tolerance to random amino acid change. Proc. Natl. Acad. Sci. U.S.A. 101, 9205-9210. doi: 10.1073/pnas.0403255101

Holmgren, A. (1976). Hydrogen donor system for Escherichia coli ribonucleosidediphosphate reductase dependent upon glutathione. Proc. Natl. Acad. Sci. U.S.A. 73, 2275-2279. doi: 10.1073/pnas.73.7.2275

Holmgren, A. (1979). Glutathione-dependent synthesis of deoxyribonucleotides. Purification and characterization of glutaredoxin from Escherichia coli. J. Biol. Chem. 254, 3664-3671.

Kim, M. K., and Kang, Y. K. (1999). Positional preference of proline in alphahelices. Protein Sci. 8, 1492-1499. doi: 10.1110/ps.8.7.1492

Kim, S. G., Chung, J. S., Sutton, R. B., Lee, J. S., Lopez-Maury, L., Lee, S. Y., et al. (2012). Redox, mutagenic and structural studies of the glutaredoxin/arsenate reductase couple from the cyanobacterium Synechocystis sp. PCC 6803. Biochim. Biophys. Acta 1824, 392-403. doi: 10.1016/j.bbapap.2011.11.012

Kumar, P., and Bansal, M. (2012). HELANAL-Plus: a web server for analysis of helix geometry in protein structures. J. Biomol. Struct. Dyn. 30, 773-783. doi: 10.1080/07391102.2012.689705

Li, R., Haile, J. D., and Kennelly, P. J. (2003). An arsenate reductase from Synechocystis sp. strain PCC 6803 exhibits a novel combination of catalytic characteristics. J. Bacteriol. 185, 6780-6789. doi: 10.1128/JB.185.23.67806789.2003

Lopez-Maury, L., Florencio, F. J., and Reyes, J. C. (2003). Arsenic sensing and resistance system in the cyanobacterium Synechocystis sp. strain PCC 6803. J. Bacteriol. 185, 5363-5371. doi: 10.1128/JB.185.18.5363-5371.2003

Lundstrom-Ljung, J., and Holmgren, A. (1995). Glutaredoxin accelerates glutathione-dependent folding of reduced ribonuclease A together with protein disulfide-isomerase. J. Biol. Chem. 270, 7822-7828. doi: $10.1074 /$ jbc. 270.14 .7822
Muller, W., Weber, H., Meyer, F., and Weissmann, C. (1978). Site-directed mutagenesis in DNA: generation of point mutations in cloned beta globin complementary dna at the positions corresponding to amino acids 121 to 123. J. Mol. Biol. 124, 343-358. doi: 10.1016/0022-2836(78)90303-0

Schrodinger, LLC. (2013). The PyMOL Molecular Graphics System, Version 1.6.

Winn, M. D., Ballard, C. C., Cowtan, K. D., Dodson, E. J., Emsley, P., Evans, P. R., et al. (2011). Overview of the CCP4 suite and current developments. Acta Crystallogr. D Biol. Crystallogr. 67, 235-242. doi: 10.1107/S0907444910045749

Worth, C. L., Preissner, R., and Blundell, T. L. (2011). SDM-a server for predicting effects of mutations on protein stability and malfunction. Nucleic Acids Res. 39, W215-W222. doi: 10.1093/nar/gkr363

Conflict of Interest Statement: The authors declare that the research was conducted in the absence of any commercial or financial relationships that could be construed as a potential conflict of interest.

Received: 28 August 2013; paper pending published: 13 September 2013; accepted: 26 October 2013; published online: 15 November 2013.

Citation: Knaff DB and Sutton RB (2013) Utility of Synechocystis sp. PCC 6803 glutaredoxin $A$ as a platform to study high-resolution mutagenesis of proteins. Front. Plant Sci. 4:461. doi: 10.3389/fpls.2013.00461

This article was submitted to Plant Physiology, a section of the journal Frontiers in Plant Science.

Copyright (c) 2013 Knaff and Sutton. This is an open-access article distributed under the terms of the Creative Commons Attribution License (CC BY). The use, distribution or reproduction in other forums is permitted, provided the original author(s) or licensor are credited and that the original publication in this journal is cited, in accordance with accepted academic practice. No use, distribution or reproduction is permitted which does not comply with these terms. 